\title{
Evaluating an antimicrobial stewardship programme implemented in an intensive care unit of a large academic hospital, using the RE-AIM framework
}

\author{
B E Nkosi, ${ }^{1} \mathrm{PhD}$; S Sibanda, ${ }^{1,2} \mathrm{PhD}$ \\ ${ }^{1}$ Department of Health Studies, Faculty of Health Sciences, University of South Africa, Pretoria, South Africa \\ ${ }^{2}$ School of Applied Social Studies, University of Bedfordshire, Luton, UK
}

Corresponding author: B E Nkosi (mabingos@live.com)

\begin{abstract}
Background. The threat of antimicrobial resistance driven by inappropriate and unnecessary use of antimicrobials is a global issue of great concern. Evidence-based approaches to optimising antimicrobial prescribing to improve patient care while reducing the rate of antimicrobial resistance continue to be implemented worldwide. However, the successes or failures of implementation of such approaches are seldom evaluated.

Objectives. To evaluate the impact of an implemented antimicrobial stewardship programme (ASP) in reducing the spread of antimicrobial resistance in the intensive care unit (ICU) of a large academic hospital using the RE-AIM framework.

Methods. A descriptive quasi-experimental study was conducted with adult patients who had been admitted to the ICU of an academic hospital in Johannesburg, South Africa. Data were extracted from patients' records using a structured questionnaire. Descriptive statistics of four RE-AIM dimensions (reach, effectiveness, adoption and implementation) and the overall impact of the implemented antimicrobial stewardship programme were calculated.

Results. From the 59 participant records, 21 patients (35.6\%) developed hospital-acquired infections and all were prescribed antimicrobials during their stay in the ICU. Twenty-seven pathogens (bacterial species) were isolated from samples acquired from the patients, including Staphylococcus aureus $(n=6 ; 22.2 \%)$, Escherichia coli $(n=4 ; 14.8 \%)$, Acinetobacter baumannii $(n=4 ; 14.8 \%)$ and Streptococcus pnuemoniae $(n=3 ; 11.11 \%)$, as well as 10 other bacterial species (37.0\%) including Corynebacterium species, Enterococcus faecium, Haemophilus influenzae, Klebsiella species, Clostridium difficile and Salmonella species. Of the 27 pathogens isolated, 19 (70.4 \%) were resistant to the prescribed antimicrobials. The overall impact of the ASP implemented in the studied facility was $67.2 \%$.

Conclusions. An ASP requires both thorough implementation and leadership support to have an impact in the reduction of antimicrobial resistance. Lack of leadership support poses a significant challenge to sustainability. There is an urgent need for behavioural change in hospital leadership.
\end{abstract}

S Afr Med J 2021;111(8):777-782. https://doi.org/10.7196/SAMJ.2021.v111i8.15363

Antimicrobial resistance is a growing global health concern that warrants attention and immediate action. ${ }^{[1]}$ Since their introduction, antimicrobial agents have effectively decreased morbidity and mortality caused by infectious diseases. Regrettably, their inappropriate and irrational use has led to an increased risk of adverse drug effects and the development of antimicrobial resistance, which renders some antimicrobials ineffective. ${ }^{[2,3]}$ Moreover, a discrepancy in diagnostic procedures vital to correctly identifying different pathogens aggravates the situation. ${ }^{[2]}$

Since healthcare settings are associated with the highest emergence and spread of antimicrobial resistance, ${ }^{[4]}$ the Infectious Diseases Society of America ${ }^{[5]}$ published guidelines recommending that all hospitals develop institutional programmes to enhance antimicrobial stewardship and hence curb the growing threat of antimicrobial resistance. Subsequently, policies and evidence-based interventions, such as antimicrobial stewardship programmes (ASPs), have been drafted and implemented in various healthcare institutions worldwide. ${ }^{[6,7]}$ Antimicrobial stewardship (AMS) refers to a collection of strategies and tools to improve clinical outcomes and use of antimicrobials, whereby selection of the optimal regimen, dose, duration and route of administration is promoted. ${ }^{[8]}$

AMS programmes are considered an important strategy in the fight against antimicrobial resistance. ${ }^{[8]}$ Dyar et al. ${ }^{[9]}$ define AMS as a coherent set of actions that promote using antimicrobials in a way that ensures sustainable access to effective therapy for all who need it. Establishing the impact of an implemented ASP in a healthcare facility is important to inform decisions about the future of the programme, whether it needs scaling up or not. ${ }^{[10]}$ From evidence, impact evaluation studies conducted on the effectiveness of the ASP are often limited to individual impact rather than the assessment of both individual and organisational impact. ${ }^{[11-14]}$ Furthermore, comprehensive impact evaluation of health interventions depends on the effectiveness of the programme/intervention, its reach in the population, and the extent to which it is properly implemented. ${ }^{[15]}$

Applying the RE-AIM framework ${ }^{[16]}$ makes it possible to comprehensively explore five different impact dimensions that affect the ASP: (i) reach for assessing the target population accomplished; (ii) effectiveness, i.e. measuring the patient outcomes resulting from the programme; (iii) adoption, i.e. measuring the number of target settings participating in the study and staff who are responsible for the management of the programme in the facility; (iv) implementation, i.e. consistency and cost of delivery; and $(v)$ maintenance of the intervention's positive effects over time. ${ }^{[16]}$ The RE-AIM framework is useful for determining which interventions work in real-world settings and assesses impact at individual and organisational levels. ${ }^{[17]}$ Although there is evidence of the effectiveness of ASPs in the 
literature, the scope of evaluation is narrow and limited to hospital length of stay, readmission, re-infection, mortality and emergence of resistance, ${ }^{[1]}$ and also economic effects of inappropriate use of antimicrobials. ${ }^{[2]}$ To our knowledge, there is a dearth of literature on comprehensive evaluation of ASPs integrated into routine use in South African (SA) hospitals.

\section{Objectives}

To advance understanding of the factors that influence the successful implementation and effectiveness of an ASP in limiting the spread of antimicrobial resistance in hospitals, a descriptive quasi-experimental study guided by the RE-AIM framework was conducted to inform best practice.

\section{Methods \\ Study design and sampling}

A descriptive quantitative quasi-experimental study of the quality of antimicrobial prescribing was undertaken in an adult intensive care unit (ICU) of a large academic hospital in Johannesburg, SA, over a 3-month period from June 2017 to August 2017. The hospital has $\sim 3200$ beds, and the average length of stay is 7.9 days. Systematic random sampling was used to identify study sites from a list of tertiary and academic hospitals in Gauteng Province. From this process, two academic hospitals were selected, and of these only one public hospital agreed to participate in this study.

The sample comprised 65 adult patients ( $\geq 18$ years) who were admitted to the ICU and had received the study recruitment letter.

\section{Study procedures}

The study recruitment letter was shared with eligible patients at the participating hospital. An invitation letter was also sent to the ICU head and ASP team, as they were to complete the institutional capacity evaluation questionnaire. Participation in the study was voluntary, and all patients and/or their families were asked to provide written consent prior to data extraction from the patient's hospital records. Participants were assured of confidentiality and anonymity, and were also told of the option to withdraw at any stage of the study.

\section{Measures}

A paper-based questionnaire was used to collect patients' data from their hospital records. The questionnaire development was informed by reviewed literature, existing surveys and published guides pertaining to the ASP. ${ }^{[5,18,19]}$ The reliability of the data collection instrument was tested prior to use, and this considered the item total correlation, inter-item correlation and Cronbach's alpha coefficient. The developed questionnaire consisted of three phases with a total of 41 items. The first phase included 11 items designed to collect information concerning the appropriateness of antimicrobials prescribed in the ICU and patients' demographic characteristics. The second phase included 7 items concerning the outcome of the patients treated with antimicrobials. The third phase included 23 items on the capacity of the hospital to prescribe antimicrobials appropriately. The questionnaire items were closed-ended but offered an opportunity for detailed responses. ${ }^{[20]}$

The RE-AIM framework dimensions were defined and calculated and formed the basis of the overall institutional evaluation of the ASP. Reach of the programme was calculated as the number of willing and participating patients divided by the total number of eligible patients whose records were available for analysis. ${ }^{[21]}$

Effectiveness of the impact of the ASP on important patient outcomes was also measured at an individual level. This involved assessment of all outcomes that resulted from the use of antimicrobials. The number of patients who developed antimicrobial resistance was expressed as a percentage of the total participants.

Adoption was assessed as an organisational measure of representativeness and characteristics of participating institution settings. The adoption dimension calculated the hospitals that participated in the study as a percentage of eligible hospitals. ${ }^{[22]}$

The implementation dimension evaluates the extent to which the ASP is delivered as intended at the organisational level. ${ }^{[16]}$ This was achieved by assessing the steps taken to prescribe appropriate antimicrobials as specified in guidelines. ${ }^{[23]}$ The core elements of hospital antibiotic stewardship programmes ${ }^{[18]}$ (leadership commitment, accountability, drug expertise, action, tracking, reporting and education) were assessed and scores were provided for each core element as follows: deficient $(0$ $25 \%)$, low (26 - 50\%), sufficient (51-75\%) or satisfactory (76-100\%).

The maintenance dimension was excluded because the duration of the collection of data was $<6$ months, the period required to measure the long-term effects of a programme.

Subsequent to the evaluation of each of the RE-AIM dimensions, the potential effectiveness of the implemented ASP was assessed. The total scores of each RE-AIM dimension were added and divided by 5 . The results thereof was considered to reflect the potential impact of the ASP. ${ }^{[24]}$

\section{Ethical considerations}

The study received ethical approval from the Higher Degrees Research Ethics Committee of the Department of Health Studies at the University of South Africa, Pretoria (ref. no. HSHDC/605/2017).

\section{Statistical analysis}

Data were analysed using SPSS version 24 (IBM, USA). Descriptive statistics were used to summarise responses to the questionnaire questions. Data relating to demographic characteristics of participants, susceptibility of bacterial isolates, processes of antimicrobial prescribing, capacity and impact measures of the ASP were presented as frequencies and percentages. Means and standard deviations were used to summarise continuous variables. Multivariate logistical regression analysis was used to establish the relationship between risk factors and hospital-acquired infections. A confidence level of $95 \%$ was used in calculations.

\section{Results}

\section{Demographic characteristics}

A total of of 59 respondents had complete records and were included in the study, with the majority (57.6\%) in the 19 - 38-year age group; males comprised $55.9 \%$ and black Africans $81.4 \%$, 52.5\% were trauma patients, $45.8 \%$ had moderate severity of illness, and $67.8 \%$ had indications for surgical prophylaxis (Table 1).

\section{Effectiveness of the ASP}

The majority of the biological specimens cultured (74.6\%) were positive for microbial agents. Two patients were admitted because of infections, and 21 developed hospital-acquired infections (HAIs); all were prescribed antimicrobials during their stay in the ICU.

Table 2 shows the susceptibility results of the pathogens isolated from the samples of patients admitted to the ICU. Among the 21 patients with HAIs, 27 pathogens were isolated. The most frequent bacterial species isolated was Staphylococcus aureus ( $n=6$; $22.2 \%)$, followed by Escherichia coli $(n=4 ; 14.8 \%)$, Acinetobacter baumannii $(n=4 ; 14.8 \%)$ and Streptococcus pnuemoniae $(n=3$; $11.1 \%)$. The least frequently isolated pathogens, all with single 
isolates (3.7\%), included Corynebacterium species, Enterococcus faecium, Haemophilus influenzae, Klebsiella species, Clostridium difficile and Salmonella species. Of the 27 isolated pathogens, 19 (70.4\%) were resistant to the prescribed antimicrobials. All 4 A. baumannii isolates (14.8\%), 4 (66.7\%) of the S. aureus and 50\% of the E. coli were resistant.

\begin{tabular}{|c|c|}
\hline Variable & \\
\hline \multicolumn{2}{|l|}{ Gender, $n(\%)$} \\
\hline Male & $33(55.9)$ \\
\hline Female & $26(44.1)$ \\
\hline \multicolumn{2}{|l|}{ Age (years) } \\
\hline Mean (SD) & $41.51(16.6)$ \\
\hline $19-38, n(\%)$ & $34(57.6)$ \\
\hline $39-58, n(\%)$ & $16(27.1)$ \\
\hline $59-78, n(\%)$ & $7(11.9)$ \\
\hline $79-98, n(\%)$ & $2(3.4)$ \\
\hline \multicolumn{2}{|l|}{ Race, $n(\%)$} \\
\hline Black African & $48(81.4)$ \\
\hline Coloured & $3(5.1)$ \\
\hline White & $6(10.2)$ \\
\hline Indian & $2(3.4)$ \\
\hline \multicolumn{2}{|l|}{ Diagnosis grouping, $n$ (\%) } \\
\hline Infectious diseases & $2(3.4)$ \\
\hline Genitourinary system & $4(6.8)$ \\
\hline Respiratory system & $6(10.2)$ \\
\hline Digestive and liver & $11(18.6)$ \\
\hline Trauma & $31(52.5)$ \\
\hline Circulatory system & $2(3.9)$ \\
\hline Endocrine, nutritional and metabolic & $3(5.1)$ \\
\hline \multicolumn{2}{|l|}{ Severity of illness, $n(\%)$} \\
\hline Minor & $13(22.0)$ \\
\hline Moderate & $27(45.8)$ \\
\hline Major & $18(30.6)$ \\
\hline Catastrophic & $6(10.2)$ \\
\hline \multicolumn{2}{|l|}{ Indication of treatment, $n(\%)$} \\
\hline Medical prophylaxis & $16(27.1)$ \\
\hline Surgical prophylaxis & $40(67.8)$ \\
\hline Therapeutic & $3(5.1)$ \\
\hline
\end{tabular}

\section{Adoption of the ASP}

There were 8 eligible academic hospitals in Gauteng Province; of these, 2 were recruited for the study, but only 1 agreed to participate.

The recommended number of trained and educated multidisciplinary ASP core members for implementation of the programme is 6. In the present study, 5 AMS members (83.3\%), including an infection prevention and protection physician, a microbiological laboratory specialist (leader), a clinician with an interest in infection and a pharmacist with expertise in infection, were identified (with the exception of nursing staff). There was evidence of the availability of local antimicrobial guidelines. The microbiological laboratory was situated in the enclosure of the healthcare facility for timely reporting of the results.

\section{Implementation of the ASP}

The results for the implementation dimension are shown in Table 3. The majority $(n=10 ; 71.4 \%)$ of the steps for prescribing quality antimicrobials were delivered as intended, with the exception of 4 steps $(28.6 \%)$. Most patients $(n=57 ; 96.6 \%)$ were administered antimicrobials intravenously.

The capacity measures for prescribing antimicrobials are presented in Table 4 . The capacity measure factoring in seven parameters was calculated to be $69.2 \%$, indicative of a sufficient capacity to prescribe quality antimicrobials. The leadership component scored the lowest at $50.0 \%$.

\section{RE-AIM impact outcome measure}

Table 5 shows the performance measures of the ASP for each REAIM dimension. For the reach dimension, a high performance rate of $90.8 \%$ was observed. For the effectiveness dimension, the programme performed fairly well, with $56.8 \%$ of patients not developing HAI, indicating some success in preventing the development of HAI. The ASP in the studied facility had a high adoption rate of $83.3 \%$, supported by the in-house microbiological laboratory, with a comprehensive list of essential antimicrobials and antimicrobial prescribing guidelines. There was a $71.4 \%$ success rate in following the steps recommended for prescribing quality and effective antimicrobials. The implementation rate of the ASP in the hospital overall was classed as good.

\section{Discussion}

ASPs are an important strategy in the fight against antimicrobial resistance. ${ }^{[8]}$ There is a dearth of literature evaluating the impact of the ASP, an important step in effective and sustainable implementation

Table 2. Frequencies and susceptibility of bacterial species isolated from microbiological samples in intensive care unit inpatients $(N=27)$

\begin{tabular}{llll}
\hline Pathogen & Resistant, $\boldsymbol{n}(\%)$ & Sensitive, $\boldsymbol{n}(\%)$ & Total, $\boldsymbol{n}(\%)$ \\
\hline Acinetobacter baumannii & $4(14.8)$ & - & $4(14.8)$ \\
Clostridium difficile & - & $1(3.7)$ & $1(3.7)$ \\
Clostridium spp. & - & $2(7.4)$ & $2(7.4)$ \\
Corynebacterium & $1(3.7)$ & - & $1(3.7)$ \\
Escherichia coli & $2(7.4)$ & $2(7.4)$ & $4(14.8)$ \\
Enterococcus faecium & $1(3.7)$ & - & $1(3.7)$ \\
Enterobacter spp. & $2(7.4)$ & - & $2(7.4)$ \\
Haemophilus influenzae & $1(3.7)$ & - & $1(3.7)$ \\
Klebsiella & $1(3.7)$ & - & $1(3.7)$ \\
Staphylococcus aureus & $4(14.8)$ & $2(7.4)$ & $6(22.2)$ \\
Streptococcus pneumoniae & $2(7.4)$ & $1(3.7)$ & $3(11.1)$ \\
Salmonella & $1(3.7)$ & - & $1(3.7)$ \\
Total & $19(70.4)$ & $8(29.6)$ & 27
\end{tabular}




\begin{tabular}{|c|c|c|}
\hline Process & Performed & Skipped \\
\hline Indication for antimicrobial use & $\checkmark$ & \\
\hline Obtain cultures & $\checkmark$ & \\
\hline \multicolumn{3}{|l|}{ Antimicrobial choice } \\
\hline Target the most likely pathogen & $\checkmark$ & \\
\hline Assess likelihood of resistance & $\checkmark$ & \\
\hline Review contraindication (allergy) & & $\checkmark$ \\
\hline Select antimicrobial with adequate tissue penetration & $\checkmark$ & \\
\hline Aim for a single antimicrobial with desired spectrum & $\checkmark$ & \\
\hline Appropriate antimicrobial dosage & $\checkmark$ & \\
\hline Appropriate dose frequency & $\checkmark$ & \\
\hline Appropriate route & $\checkmark$ & \\
\hline Therapeutic drug monitoring & & $\checkmark$ \\
\hline Desired spectrum covered & $\checkmark$ & \\
\hline \multicolumn{3}{|l|}{ De-escalation } \\
\hline Route & & $\checkmark$ \\
\hline Spectrum & & $\checkmark$ \\
\hline Total, $n(\%)$ & $10(71.4)$ & $4(28.6)$ \\
\hline
\end{tabular}

\section{Table 4. Capacity measures of antimicrobial stewardship programme}

\begin{tabular}{llllll}
\hline Key elements & Yes, $\boldsymbol{n}$ & No, $\boldsymbol{n}$ & Total, $\boldsymbol{n}$ & $\%$ & Characterised \\
\hline Leadership & 1 & 1 & 2 & 50 & Low \\
Accountability & 1 & 0 & 1 & 100 & Satisfactory \\
Drug expertise & 5 & 2 & 17 & 71.4 & Sufficient \\
Action & 11 & 6 & 8 & 7.7 & Sufficient \\
Tracking & 6 & 2 & 3 & 66.7 & Sufficient \\
Reporting & 2 & 1 & 1 & 100 & Sufficient \\
Education & 1 & 0 & 39 & 69.2 & Satisfactory \\
Total & 27 & 12 & & & Sufficient
\end{tabular}

Table 5. Impact measures of RE-AIM dimensions

\begin{tabular}{|c|c|c|c|}
\hline Dimension & Description & Evaluation criteria & Evaluation \\
\hline \multirow[t]{3}{*}{ Reach } & \multirow{3}{*}{$\begin{array}{l}\text { Ratio of participating ICU patients to number of } \\
\text { patients recruited }\end{array}$} & Eligible patients recruited & $n=56$ \\
\hline & & Participating patients & $n=59$ \\
\hline & & Performance rate & $90.7 \%$ \\
\hline \multirow[t]{3}{*}{ Effectiveness } & \multirow{3}{*}{$\begin{array}{l}\text { Ratio of inpatients who developed HAI to number of } \\
\text { patients tested for HAI }\end{array}$} & Biological samples tested & $n=44$ \\
\hline & & HAI-positive samples & $n=19$ \\
\hline & & Performance rate & $56.8 \%$ \\
\hline \multirow[t]{7}{*}{ Adoption } & \multirow{7}{*}{$\begin{array}{l}\text { Organisational measure of the number and } \\
\text { representativeness of staff and settings who have } \\
\text { adopted the programme, as well as institutional } \\
\text { support in programme delivery }\end{array}$} & Eligible recruited facilities & $n=2$ \\
\hline & & Participating facilities & $n=1$ \\
\hline & & Settings & $50.0 \%$ \\
\hline & & ASP team members & $n=5$ \\
\hline & & Recommended ASP team members & $n=6$ \\
\hline & & Adoption rate & $83.3 \%$ \\
\hline & & ASP staff & $66.7 \%$ \\
\hline \multirow[t]{5}{*}{ Implementation } & \multirow{5}{*}{$\begin{array}{l}\text { Organisational measure of the resources and the extent } \\
\text { to which ASP was delivered as intended }\end{array}$} & Steps taken for prescribing antimicrobials & $n=10$ \\
\hline & & $\begin{array}{l}\text { Steps required for prescribing } \\
\text { antimicrobials }\end{array}$ & $n=14$ \\
\hline & & Processes success rate & $71.4 \%$ \\
\hline & & Core elements of ASP & $69.2 \%$ \\
\hline & & Fidelity rate & $70.3 \%$ \\
\hline Average impact & $(\mathrm{R}+\mathrm{E}+\mathrm{A}+\mathrm{IM}) / 4$ & ASP impact & $71.1 \%$ \\
\hline
\end{tabular}


of the programme. The present study describes the application of the RE-AIM framework in evaluation of the impact of an ASP in reducing the spread of antimicrobial resistance in Gauteng, SA. Guided by the RE-AIM framework, four dimensions were considered for the impact of the ASP: reach and effectiveness were analysed at the individual level, whereas adoption and implementation were assessed at the organisational level. Overall, the impact of the ASP in limiting the spread of antimicrobial resistance was significantly sufficient.

The programme reached $\sim 90 \%$ of eligible patients admitted to the ICU. This was exceptional considering the barriers to recruiting critically ill patients and distrust of researchers concerning confidentiality of private information. Reach was substantially greater for black African patients than for other ethnic groups, and this could be attributed to more black and coloured people using public health facilities compared with the white and Indian population, who mainly use private health facilities. ${ }^{[25]}$

Most patients whose records were reviewed were $<65$ years of age. This was a surprising finding, as the global population is ageing, so an increase in admission of elderly patients to ICUs would be expected. ${ }^{[26]}$ Furthermore, the elderly are commonly admitted to the ICU as a result of conditions that result in a compromised immune system, such as organ and tissue dysfunction and underlying chronic diseases. ${ }^{[27]}$

The adoption rates in the present study were limited to the one of the eight eligible academic hospitals that fully participated. The other hospitals therefore missed the opportunity to assess the comprehensive impact of the ASP implemented in their institution, and therefore to determine the problematic areas. To improve on the performance of an ASP, it is recommended that the ASP team should include, at a minimum, a physician, a pharmacist, a clinical microbiologist and an infection prevention and protection physician, with at least one member having experience in AMS. ${ }^{[5,28]}$ The participating facility in this study had five healthcare professionals as core members of ASP team, i.e. an infection prevention and protection physician, a microbiological laboratory specialist (leader), clinicians with an interest in infection, and a pharmacist with expertise in infection. However, the composition of the core team members may vary. In the UK, for example, where infection specialist experts are utilised, AMS is viewed as the responsibility of all healthcare professionals, not just the ASP team. ${ }^{[28]}$

Adherence to the guidelines of prescribing antimicrobials in the healthcare facility is imperative so as to limit the emergence of antimicrobial resistance. The present study reported a high threshold in adhering to the recommendations for prescribing antimicrobials, suggesting that a remarkable amount of care is devoted to ensuring effective and responsible prescribing of antimicrobials to reduce antimicrobial resistance. Moreover, there is a strong association between adherence to antimicrobial prescribing guidelines and prudent use of antimicrobials, implying that adhering to the recommended guidelines can reduce inappropriate use or overuse of antimicrobials..$^{[29]}$

The present study illustrates the use of the core elements of an ASP for assessing implementation, and suggests that the core elements adequately capture the essence of the inputs for the functioning of the programme. Overall, the inputs for the implementation of the programme were found to be sufficient at $69.2 \%$. Most core elements showed sufficient scores of maintenance (51 - 75\%), with the exception of the leadership element with a low value of $50.0 \%$, suggesting that a sufficiently sustainable programme is implemented in the hospital. Owing to low leadership support, the organisation lacks personnel and funding, which is perceived as the major barrier to a sustainable programme. Hospital leadership support is essential to the success of an ASP by ensuring that the programme has sufficient budget, technology, time management and resources. ${ }^{[30,31]}$ Furthermore, with no formal funding available for running the programme, the health institution depended on sponsorships for financing.

\section{Study limitations}

This study had some limitations. The collection of information from adult patients only means that the results are not generalisable to paediatric patients. The fact that there was only a single participating hospital may not allow the generalisation of the results to other hospital settings and the country at large.

The data collection period was not long enough to monitor the longterm outcome of the patients or determine the programme's inclusive effectiveness. A longer study duration may have demonstrated more meaningful results by showing maintenance of the patients' improvement or re-admission to the hospital and identifying the causes thereof. However, one of the strengths was that being in one centre ensured comprehensive data collection, and the results can be used in future comparative studies.

\section{Conclusions}

There is ample evidence in the literature that an effective and welladopted and implemented ASP can result in more appropriate use of antimicrobials, leading to improved patient safety and outcomes. Although the present study showed that the performance of the ASP implemented in the facility investigated was sufficient, there are strategies that can be used to optimise the programme. The study demonstrated that regular evaluation of the health programme is a necessity for identifying weaknesses and strengths in order to upscale it.

The studied facility had no adequate management support for the implemented ASP. There was no financial and personnel support to sustain the programme, and most of the healthcare workers were discouraged from participating in it, leading to inadequate performance of the programme. Leadership support is critical for the success of ASPs, and it is therefore recommended that the hospital leadership should be persuaded to be more concerned about their importance.

\section{Recommendations for future research}

Owing to the limitations of this study, future research should consider including both public and private hospitals, broadening the scope to include different types of ICUs and patients, and conducting further studies with larger sample sizes and multicentre sampling. It is further recommended that the study duration be increased, allowing follow-up studies. For a more thorough impact evaluation, the measure of cost-effectiveness (the worth of the programme) is also recommended to assess whether the benefits outweigh the cost. For a comprehensive and more robust evaluation, it is recommended that all five RE-AIM dimensions be assessed in future studies.

\section{Declaration. None.}

Acknowledgements. The authors are grateful to the institution that gave approval to conduct the study, the healthcare providers for their cooperation, and the patients who participated.

Author contributions. All authors contributed equally to aspects of the article including study design, data collection and analysis, drafting, proofreading and editing of the manuscript. 
Funding. None.

Conflicts of interest. None.

1. Eckart J, Hogan M, Mao Y, et al. Antimicrobial stewardship programs: Effects on clinical and economic outcomes and future directions. J Clin Outcomes Manag 2017;24(7):309-318. https://pdfs. semanticscholar.org/c526/666f6f128c25846d7dd188c88682546ba6c9.pdf (accessed 15 August 2016).

2. Dik J-WH, Poelman R, Friedrich AW, et al. An integrated stewardship model: Comprising antimicrobial, infection prevention and diagnostic stewardship (AID Stewardship). Future Microbiol 2015;11(1):93-102. https://doi.org/10.1128/JCM.01283-17

3. Nia SS, Hiremath SRR, Prasad S. Assessment of antimicrobial use pattern using World Health Organization prescribing indicators at a tertiary hospital: A prospective, observational study. J App Pharm Sci 2018;8(06):132-138. https://doi.org/10.7324/JAPS.2018.8617

4. Pinder RJ, Sallis D, Berry A, Chadborn T. Antibiotic prescribing and behaviour change in healthcare setting: Literature review and behavioural analysis. London, UK: Department of Health and Public Health England, 2015. https://www.gov.uk/government/publications/antibiotic-prescribing-andbehaviour-change-in-healthcare-settings (accessed 23 March 2016).

5. Dellit TH, Owens RC, McGowan JE jr, et al. Infectious Diseases Society of America and the Society for Healthcare Epidemiology of America guidelines for developing an institutional program to enhance Healthcare Epidemiology of America guidelines for developing an institutional program to en
antimicrobial stewardship. Clin Infect Dis 2007;44(2):159-177. https://doi.org/10.1086/510393

6. James R, Luu S, Avent M, et al. A mixed methods study of the barriers and enablers in implementing James R, Luu S, Avent M, et al. A mixed methods study of the barriers and enablers in implementing
antimicrobial stewardship programmes in Australian regional and rural hospitals. J Antimicrob antimicrobial stewardship programmes in Australian regional and

7. Charani E, Castro-Sanchez E, Sevdalis N, et al. Understanding the determinants of antimicrobial prescribing within hospitals: The role of 'prescribing etiquette.' Clin Infect Dis 2013;57(2):188-186. https://doi.org/10.1093/cid/cit21

8. Osthoff M, Bielicki J, Widmer AF, et al. Evaluation of existing and desired antimicrobial stewardship activities and strategies in Swiss hospitals. Swiss Med Wkly 2017;147:w14512. https://doi.org/10.4414/ smw.2017.14512

9. Dyar OJ, Huttner B, Schouten J, Pulcini C; on behalf of ESGAP. What is antimicrobial stewardship? Clin Microbiol Infect 2017;23(11):793-798. https://doi.org/10.1016/j.cmi.2017.08.026

10. Gertler PJ, Martinez S, Premand P, Rawlings, LB, Vermeersch CMJ. Impact Evaluation in Practice. 2nd ed. Washington, DC: International Bank for Reconstruction and Development/World Bank, 2016. https://openknowledge.worldbank.org/bitstream/handle/10986/25030/9781464807794. Bank, 2016. https://openknowledge. worldbank.org/bitstre

11. Chang Y-Y, Chen H-P, Lin C-W, et al. Implementation and outcomes of an antimicrobial stewardship program: Effectiveness of education. J Chin Med Assoc 2017;80(6):253-359. https://doi.org/10.1016/j. program: Effective

12. Kaki R, Elligsen $M$, Walker S, et al. Impact of antimicrobial stewardship in critical care: A systematic review. J Antimicrob Chemother 2011;66(6):1223-1230. https://doi.org/10.1093/jac/dkr13

13. Filice G, Drekonja D, Greer N, et al. Antimicrobial stewardship programs in inpatient settings A systematic review. VA-ESP Project \#09-099, 2013. https://www.hsrd.research.va.gov/publications/ esp/antimicrobial.pdf (accessed 16 June 2016).

14. Karanika S, Paudel S, Grigoras C, et al. Systematic review and meta-analysis of clinical and economic outcomes from the implementation of hospital-based antimicrobial stewardship programs. Antimicrob Agents Chemother 2016;60(8):4840-4852. https://doi.org/10.1128/AAC.00825-16

15. Fernandez ME, ten Hoor G A, van Lieshout $\mathrm{S}$, et al. Implementation mapping: Using implementation mapping to develop implementation strategies. Front Public Health 2019:7.158 https./1dol $\operatorname{org} / 10.3389 /$ fpubh 2019.00158
16. Wozniak L, Rees S, Soprovich A, et al. Applying the RE-AIM framework to the Alberta's Caring for Diabetes Project: A protocol for a comprehensive evaluation of primary care quality improvement interventions. BMJ Open 2012;2:e002099. https://doi.org/10.1136/bmjopen-2012-002099

17. National Collaborating Centre for Methods and Tools (NCCMT). Assessing the public health impact of health promotion initiatives: RE-AIM evaluation framework. Hamilton, Ont.: McMaster University, 2010. www.nccmt.ca/knowledge-repositories/search/70 (accessed 11 October 2018)

18. Centers for Disease Control and Prevention. Core elements of hospital antibiotic stewardship programs. Atlanta, Ga.: US Department of Health and Human Services, 2014. www.cdc.gov/ getsmart/healthcare/implementation/core-elements.html (accessed 8 July 2018).

19. Public Health England. Start smart - then focus: Antimicrobial stewardship toolkit for English hospitals. 17 November 2011. https://www.gov.uk/government/publications/english-start smartthen focus-espaur-report (accessed 15 August 2018).

20. Siniscalco MT, Auriat N. Questionnaire design. In: Ross KN, ed. Module 8, Quantitative research method in education planning. International Institute for Educational Planning/UNESCO, 2005. http://dl.icdst.org/pdfs/files/2cdf41a15035f773351ebd3092d7eeaa.pdf (accessed 25 September http:/ 2018

21. Santos SZ, Tagai EK, Scheirer MA, et al. Adoption, reach, and implementation of a cancer education intervention in African American churches. Implement Sci 2017;12:36. https://doi.org/10.1186/ 13012-017-0566-2

22. Ory MG, Altpeter M Belza B, et al. Perceptions about community applications of RE-AIM in the promotion of evidence-based programs for older adults. Eval Health Prof 2015;38(1):15-20. https:// doi.org/10.1177/0163278714542335

23. Wasserman S, Boyles T, Mendelson M. A pocket guide to antibiotic prescribing for adults in South Africa 2015. South African Antibiotic Stewardship Programme, 2014. https://www.docweb.co.za/ docweb-files/Antibiotic\%20Guidelines\%20Article.pdf (accessed 20 November 2018).

24. Compernolle S, de Cocker K, Lakerveld J, et al. A RE-AIM evaluation of evidence-based multilevel interventions to improve obesity-related behaviours in adults: A systematic review (the SPOTLIGHT interventions to improve obesity-related behaviours in adults: A systematic review (the SPOT-1
project). Int J Behav Nutr Phys Act 2014:11:147. https://doi.org/10.1186/s12966-014-0147-3

25. Lehohla P. Use of health facilities and levels of selected health conditions in South Africa: Findings from the general household survey, 2011. Pretoria: Statistics South Africa, 2013. http://www.statssa. gov.za/publications/Report-03-00-05/Report-03-00-052011.pdf (accessed 20 November 2018)

26. Solis-Vernandez PS, Vidales-Reyes M, Gorza-Gonzalez E, et al. Hospital-acquired infections in elderly versus younger patients in an acute care hospital. Int J Infect 2016;3(1):e32620. https://doi. org/10.17795/iji-32620

27. Ozdermir K, Dizbay M. Nosocomial infection and risk factors in elderly patients in intensive care units. J Microbiol Infect Dis 2015;5(1):38-43. https://doi.org/10.5799/ahinjs.02.2015.01.0714

28. Trivedi KK, Dumartin C, Gilchrist M, et al. Identifying best practices across three countries: Hospital antimicrobial stewardship in the United Kingdom, France, and the United States. Clin Infect Dis 2014;59(Suppl 3):170-178. https://doi.org/10.1093/cid/ciu538

29. Elias C, Moja L, Mertz D, et al. Guideline recommendations and antimicrobial resistance: The need for a change. BMJ Open 2017;7:e016264. https://doi.org/10.1136/bmjopen-2017-016264

30. National Quality Forum. National quality partners' playbook: Antibiotic stewardship acute care. Washington, DC: NQF, 2016. https://www.ruralcenter.org/resource-library/national-qualitypartners-playbook-antibiotic-stewardship-in-acute-care (accessed 26 July 2017)

31. Pollack LA, Srinivasan A. Core elements of hospital antibiotic stewardship programs from the Centers for Disease Control and Prevention. Clin Infect Dis 2014;59(3):S97-S100. https://doi. org/10.1098/cid/ciu542

Accepted 16 January 2021. 